\title{
Integrability and Multiple Limit Cycles in a Predator-Prey System with Fear Effect
}

\author{
Xinli Li ${ }^{1}{ }^{1}$ and Ming Zhang $\mathbb{D D}^{2}$ \\ ${ }^{1}$ Logistic School, Linyi University, Linyi 276000, China \\ ${ }^{2}$ College of Information Science and Engineering, Linyi University, Linyi 276000, China \\ Correspondence should be addressed to Xinli Li; lixinli0539@sina.com and Ming Zhang; zhangming@lyu.edu.cn
}

Received 22 February 2019; Accepted 11 June 2019; Published 27 June 2019

Academic Editor: Gen Q. Xu

Copyright (c) 2019 Xinli Li and Ming Zhang. This is an open access article distributed under the Creative Commons Attribution License, which permits unrestricted use, distribution, and reproduction in any medium, provided the original work is properly cited.

In this paper, a class of predator-prey systems with fear effect is investigated, the integrability conditions of the origin and the positive equilibrium are obtained, and the fact that three limit cycles can be bifurcated from the positive equilibrium is proved, so bistable phenomenon can occur for this system.

\section{Introduction}

Predator-prey systems which were considered to be among the most important models in ecological systems have been investigated intensively and extensively since they were proposed; there have been many good results for these systems. The first model for predator-prey problem was Lotka-Volterra model; this system was improved gradually year by year. Many new models were constructed with the development of biological system.

Functional response which was introduced by Holling has a great effect on predator-prey system. In 1965, the famous Holling type II functional response of predators [1] was proposed by Holling. As application, the population dynamics of predator-prey systems with the Holling type II functional response have been studied by many authors; for such a model, the result about the existence of a unique stable limit cycle has also been proved. Considering the reality, many more complicated functional responses were suggested one by one, and predator-prey systems have also been modelled gradually. For example, some monotone response functions dependent on prey have been investigated by May [2], Seo and DeAngelis [3], and Huang et al. [4]. Furthermore, Zhu et al. [5], Ruan and Xiao [6], Freedman and Wolkowicz [7], and Wolkowicz [8] studied some nonmonotone response functions dependent on prey. Some interesting bifurcation phenomenon was found for those systems. Functional responses dependent on prey only have been considered intensively; some functional responses dependent on both prey and predators were also considered in recent years, and the famous functional responses dependent on both prey and predators are the Beddington-DeAngelis functional responses, see [9-13]), and ratio dependent functional response $[14,15]$.

Although the functional responses become more and more complicated for predator-prey systems, they can only reflect the direct killing. There is profound lack of the functional responses and many realities which cannot be concluded in those systems. For example, in 2016, Zou and Wang considered a predator-prey model incorporating the cost of fear to explore the impact that fear can have on population dynamics in predator-prey systems, dynamical behaviors of this system was discussed, and a limit cycle which can be bifurcated from the positive equilibrium by Hopf bifurcation was proved [16]. But the number of limit cycles is not considered in their paper. In fact there can be many limit cycles in predator-prey system which can lead to multiple stable phenomenons. For example, the system of Leslie-type predator-prey schemes with a nonmonotonic functional response and Allee effect allows the existence of three limit cycles under certain conditions over the parameters; by Hopf bifurcation [17], the first two cycles 
are infinitesimal ones generated, and then the third one can be bifurcated from a homoclinic loop. The singular perturbation theory which was developed by Dumortier and Roussarie can be used to study the canard phenomenon. The authors studied the canard phenomenon for predator-prey systems with response functions of Holling types [18] by using this method. For a Gause type predator-prey system with Holling type III functional response and Allee effect on prey, multiple limit cycles were considered in [19] by multiple Hopf bifurcations. In [20], a criterion to calculate the multiplicity of a multiple focus was developed by the authors for general predator-prey systems. Recently, the number of limit cycles in a Leslie-Gower-type predator-prey system with weak Allee effect on prey was considered in [21].

The number of limit cycles is closely related not only to the Hilbert 16th problem but also to bistable phenomenon or multiple stable phenomenon. For instance, in [21], the existence conditions for which three limit cycles appear and surround different equilibrium points were proved. Above all, the three limit cycles have different stability which means the phenomenon of multistability. For finitely smooth planar autonomous differential systems, in [22], the author considered the bifurcation theory. Theory of rotated equations is discussed and applied to a population model in [23]. For a family of real planar polynomial ODE systems depending on parameters, the problem of how to determine the systems in the family which become time-reversible after some affine transformation was considered in [24]. Recently, an improvement on the number of limit cycles bifurcating from a nondegenerate center of homogeneous polynomial systems was obtained in [25] by normal form method. Some new perturbation methods were given in [26-28]. The integrability of equivalent systems was also considered in [29, 30]. Therefore, in this paper, we reinvestigated the predatorprey system with fear effect which is written as follows:

$$
\begin{aligned}
& \frac{d u}{d t}=\frac{r_{0} u}{1+k v}-d u-a u^{2}-\frac{p u v}{1+q u}, \\
& \frac{d v}{d t}=\frac{p u v}{1+q u}+m v .
\end{aligned}
$$

By transformation

$$
\begin{aligned}
& \bar{u}=\frac{c p-m q}{m} u, \\
& \bar{v}=k v,
\end{aligned}
$$

and time scale

$$
d t=\frac{(1+q u)(1+k v)}{m} d \bar{t},
$$

system (1) can be reduced to

$$
\begin{aligned}
& \frac{d u}{d t} \\
& \quad=u\left(a_{1}+a_{2} u-a_{3} v-a_{4} u v-a_{5} u^{2}-a_{6} v^{2}-a_{5} u^{2} v\right), \\
& \frac{d v}{d t}=v(u-1)(1+v) .
\end{aligned}
$$

And system (4) has three singular points

$$
(0,0),\left(\frac{a_{2}+\sqrt{a_{2}^{2}+4 a_{1} a_{5}}}{2 a_{5}}, 0\right),\left(1, \bar{v}_{1}\right)
$$

where

$$
\begin{aligned}
& \bar{v}_{1} \\
& =-\frac{a_{3}+a_{4}+a_{5}-\sqrt{\left(a_{3}+a_{4}+a_{5}\right)^{2}+4\left(a_{1}+a_{2}-a_{5}\right) a_{6}}}{2 a_{6}} .
\end{aligned}
$$

The classification of the three singular points and stability have been discussed in [16], but the integrability and center problem remained. The rest of the paper is organized as follows. In the next section, we prove that the origin of system (4) is integral. In Section 3, the first four Lyapunov constants will be computed; bifurcation of limit cycles and center conditions of (4) are investigated. Section 4 is devoted to discussing the kind of the boundary equilibrium. At last, a conclusion is drawn to illustrate that bistable phenomenon can occur in this system.

\section{Integrability Conditions of $(0,0)$}

For the integrability of origin of system (4), the authors have proved that it is a saddle. In this section, we will compute the saddle values; furthermore, the integrability conditions can be obtained at the same time. We only consider a special case; namely, let $a_{1}=1$; we study the integrability conditions theoretically. The system (4) becomes

$$
\begin{aligned}
& \frac{d u}{d t}=u\left(1+a_{2} u-a_{3} v-a_{4} u v-a_{5} u^{2}-a_{6} v^{2}-a_{5} u^{2} v\right) \\
& \frac{d v}{d t}=v(u-1)(1+v)
\end{aligned}
$$

Theorem 1. The first five saddle values at the origin of system (7) are

$$
\begin{aligned}
& \mu_{1}=a_{2} a_{3}-a_{4} \\
& \mu_{2}=-a_{5}-3 a_{3} a_{5}-2 a_{3}^{2} a_{5}+a_{2} a_{6}-a_{5} a_{6}, \\
& \text { If } 1+3 a_{3}+2 a_{3}^{2}+a_{6} \neq 0 \\
& \mu_{3}=-\frac{a_{2} a_{6}}{1+3 a_{3}+2 a_{3}^{2}+a_{6}}\left(-2-5 a_{3}+2 a_{2} a_{3}-3 a_{3}^{2}\right. \\
& \left.+5 a_{2} a_{3}^{2}+3 a_{2} a_{3}^{3}+8 a_{6}+a_{2} a_{6}+9 a_{3} a_{6}+a_{2} a_{3} a_{6}\right), \\
& \text { (1) }\left(1+a_{3}\right)\left(2 a_{3}+3 a_{3}^{2}+a_{6}\right) \neq 0 \\
& \mu_{4}=-\frac{a_{6}^{2}\left(2+5 a_{3}+3 a_{3}^{2}-8 a_{6}-9 a_{3} a_{6}\right)}{3\left(1+a_{3}\right)^{2}\left(1+3 a_{3}+2 a_{3}^{2}+a_{6}\right)\left(2 a_{3}+3 a_{3}^{2}+a_{6}\right)^{3}} h_{1}, \\
& \mu_{5}=\frac{a_{6}^{2}\left(2+5 a_{3}+3 a_{3}^{2}-8 a_{6}-9 a_{3} a_{6}\right)}{24\left(1+a_{3}\right)^{3}\left(1+3 a_{3}+2 a_{3}^{2}+a_{6}\right)^{2}\left(2 a_{3}+3 a_{3}^{2}+a_{6}\right)^{4}} h_{2} \text {, }
\end{aligned}
$$




$$
\begin{gathered}
\mu_{6}=-\frac{a_{6}^{2}\left(2+5 a_{3}+3 a_{3}^{2}-8 a_{6}-9 a_{3} a_{6}\right)}{120\left(1+a_{3}\right)^{4}\left(1+3 a_{3}+2 a_{3}^{2}+a_{6}\right)^{2}\left(2 a_{3}+3 a_{3}^{2}+a_{6}\right)^{5}} \\
\quad \cdot h_{3} ;
\end{gathered}
$$

(2) $a_{3}=-1$

$$
\mu_{3}=\frac{a_{2} a_{6}}{2} ;
$$

(3) $a_{6}=-2 a_{3}-3 a_{3}^{2}$

$$
\begin{aligned}
& \mu_{3}=\frac{a_{2} a_{3}\left(2+3 a_{3}\right)^{2}}{2\left(-1-a_{3}+a_{3}^{2}\right)}\left(1+9 a_{3}+9 a_{3}^{2}\right), \\
& \mu_{4}=-\frac{a_{2} a_{3}\left(2+3 a_{3}\right)}{243\left(-1-a_{3}+a_{3}^{2}\right)}\left(72+74 a_{2}+a_{2}^{2}-144 a_{3}\right. \\
&\left.+36 a_{2} a_{3}\right), \\
& \mu_{5}=\frac{a_{2} a_{3}\left(2+3 a_{3}\right)}{48\left(-1-a_{3}+a_{3}^{2}\right)^{2}}\left(163092+310204 a_{2}\right. \\
&+142507 a_{2}^{2}+2052 a_{2}^{3}+259668 a_{3}+444930 a_{2} a_{3} \\
&\left.+179790 a_{2}^{2} a_{3}+2752 a_{2}^{3} a_{3}\right) . \\
& \text { If } a_{6}=-\left(1+3 a_{3}+2 a_{3}^{2}\right), \\
& \quad \mu_{2}=-a_{2}\left(1+a_{3}\right)\left(1+2 a_{3}\right),
\end{aligned}
$$

(4) $a_{2}=0$

$$
\begin{aligned}
& \mu_{3}=\left(1+a_{3}\right)^{2}\left(5+9 a_{3}\right) a_{5}, \\
& \mu_{4}=-\frac{1}{3}\left(1+a_{3}\right)^{2}\left(274+717 a_{3}+407 a_{3}^{2}\right) a_{5} ;
\end{aligned}
$$

(5) $a_{3}=-1 / 2$

$$
\mu_{3}=\frac{1}{8}\left(2+a_{2}\right)\left(1+a_{3}\right) a_{5} .
$$

Here $h_{1}, h_{2}, h_{3}$ are given in the appendix.

Thus, we can get the following integrability conditions of system (7) easily.

Theorem 2. The origin of system (7) is integrability if and only if one of the following conditions holds:

$$
\begin{aligned}
\text { (I) } a_{2} & =a_{4}=a_{5}=0 ; \\
\text { (II) } a_{4} & =a_{2} a_{3}, \\
& a_{5}=a_{6}=0 ;
\end{aligned}
$$

(III)

$$
\begin{aligned}
& a_{4}=-a_{2}, \\
& a_{3}=-1, \\
& a_{6}=0 ; \\
& a_{2}=-2, \\
& a_{3}=-\frac{1}{2}, \\
& a_{4}=1, \\
& a_{6}=0 .
\end{aligned}
$$$$
\text { (IV) } a_{2}=-2 \text {, }
$$

Proof. When condition (I) holds, system (7) becomes

$$
\begin{aligned}
& \frac{d u}{d t}=u\left(1-a_{3} v-a_{6} v^{2}\right), \\
& \frac{d v}{d t}=v(u-1)(1+v),
\end{aligned}
$$

which has an integrating factor

$$
\mu_{1}(u, v)=\frac{1}{u v(1+v)} .
$$

When condition (II) holds, system (7) can be rewritten as

$$
\begin{aligned}
& \frac{d u}{d t}=u\left(1+a_{2} u-a_{3} v-a_{2} a_{3} u v\right), \\
& \frac{d v}{d t}=v(u-1)(1+v),
\end{aligned}
$$

which admits an integrating factor

$$
\begin{gathered}
\frac{1}{(-1+u) u v(1+v)}, \quad a_{2}=-1, \\
(u v)^{\left(-1-2 a_{2}\right) /\left(1+a_{2}\right)}(1+v)^{\left(-1+a_{2} a_{3}\right) /\left(1+a_{2}\right)}, \quad a_{2} \neq-1 .
\end{gathered}
$$

When condition (III) holds, system (7) can be changed into

$$
\begin{aligned}
& \frac{d u}{d t}=u\left(1+a_{2} u+v+a_{2} u v-a_{5} u^{2}-a_{5} u^{2} v\right), \\
& \frac{d v}{d t}=v(u-1)(1+v),
\end{aligned}
$$

which admits an integrating factor

$$
\mu_{2}(u, v)=\frac{1}{u\left(-1-a_{2} u+a_{5} u^{2}\right) v(1+v)} .
$$

When condition (IV) holds, system (7) can be transformed into

$$
\begin{aligned}
& \frac{d u}{d t}=u\left(1-2 u+\frac{1}{2} v-u v-a_{5} u^{2}-a_{5} u^{2} v\right), \\
& \frac{d v}{d t}=v(u-1)(1+v),
\end{aligned}
$$

which has an integrating factor

$$
\mu_{3}(u, v)=\frac{1}{u^{3} v^{3}} .
$$




\section{Hopf Bifurcation at $\left(1, \bar{v}_{1}\right)$}

According to results in [16], there exists a positive singular point $\left(1, \bar{v}_{1}\right)$. Let $x=u-1, y=v-\bar{v}_{1}$; system (4) can be transformed into

$$
\begin{aligned}
& \frac{d x}{d t}=J_{11} x+J_{12} y+f_{1}(x, y), \\
& \frac{d y}{d t}=J_{21} x+J_{22} y+f_{2}(x, y) .
\end{aligned}
$$

Here

$$
\begin{aligned}
& J_{11}=a_{2}-2 a_{5}-a_{4} v_{1}-2 a_{5} v_{1} \\
& J_{12}=-a_{3}-a_{4}-a_{5}-2 a_{6} v_{1} ; \\
& J_{21}=v_{1}\left(1+v_{1}\right) \\
& J_{22}=0
\end{aligned}
$$

Suppose there are two characteristic values $\lambda_{1}=i, \lambda_{2}=-i$; then

$$
\begin{aligned}
\lambda_{1}+\lambda_{2} & =J_{11}=0, \\
\lambda_{1} \lambda_{2} & =J_{12} J_{21}=1,
\end{aligned}
$$

namely,

$$
a_{2}-2 a_{5}-a_{4} v_{1}-2 a_{5} v_{1}=0
$$

and we have

$$
\bar{v}_{1}=\frac{a_{2}-2 a_{5}}{a_{4}+2 a_{5}} .
$$

Without loss of generality, let

$$
\begin{aligned}
& a_{4}=-2 a_{5}+\beta, \\
& a_{2}=2 a_{5}+\alpha \beta,
\end{aligned}
$$

and the singular point $\left(1, \bar{v}_{1}\right)$ becomes $(1, \alpha)$. From the first equation $J_{11}=0$, we get

$$
a_{6}=\frac{a_{1}+a_{5}-a_{3} \alpha+a_{5} \alpha}{\alpha^{2}}
$$

Meanwhile, the second equation $J_{12} J_{21}=1$ means that

$$
\left(-a_{3}-a_{4}-a_{5}-2 a_{6} v_{1}\right) v_{1}\left(1+v_{1}\right)=1,
$$

which yields

$$
=\frac{2 a_{1}+2 a_{5}+2 a_{1} \alpha+3 a_{5} \alpha+a_{5} \alpha^{2}+\alpha \beta+\alpha^{2} \beta-1}{\alpha(1+\alpha)} .
$$

$$
\mu_{1}=\frac{i}{4 \alpha^{2}(1+\alpha)}\left(a_{5}\left(2+\alpha^{2}(1+\alpha) \beta\right)+\alpha(1+\alpha) \beta\left(-1+(1+\alpha)\left(a_{1}+\alpha \beta\right)\right)\right)
$$

Accordingly, system (7) can be changed into

$$
\begin{aligned}
\frac{d x}{d t} & =-\frac{1+u}{\alpha^{2}(1+\alpha)}\left(-a_{5} u^{2} \alpha^{2}(1+\alpha)^{2}\right. \\
& -v \alpha\left(1+u \alpha(1+\alpha)\left(a_{5} u+\beta\right)\right) \\
& \left.+v^{2}\left(-1+a_{1}(1+\alpha)+(1+\alpha)\left(a_{5}+\alpha \beta\right)\right)\right), \\
\frac{d y}{d t} & =u(v+\alpha)(1+v+\alpha) .
\end{aligned}
$$

The Jacobin matrix at the $(1, \alpha)$ of system (34) can be written as follows:

$$
J_{(0,0)}^{1}=\left[\begin{array}{cc}
0 & -\frac{1}{\alpha(1+\alpha)} \\
\alpha(1+\alpha) & 0
\end{array}\right] \text {, }
$$

and by linear transformation

$$
\begin{aligned}
& x=v, \\
& y=-\alpha(1+\alpha) u
\end{aligned}
$$

system can be shifted to

$$
\begin{aligned}
\frac{d u}{d t} & =-\frac{v(u+\alpha)(1+u+\alpha)}{\alpha(1+\alpha)}, \\
\frac{d v}{d t} & =-\frac{-v+\alpha+\alpha^{2}}{\alpha^{2}(1+\alpha)}\left(-u^{2}+a_{1} u^{2}+a_{5} u^{2}-a_{5} v^{2}\right. \\
& -a_{5} u v^{2}-u \alpha-u^{2} \alpha+2 a_{1} u^{2} \alpha+2 a_{5} u^{2} \alpha-a_{5} v^{2} \alpha \\
& -u \alpha^{2}+a_{1} u^{2} \alpha^{2}+a_{5} u^{2} \alpha^{2}+u^{2} \alpha \beta+u v \alpha \beta \\
& \left.+2 u^{2} \alpha^{2} \beta+u v \alpha^{2} \beta+u^{2} \alpha^{3} \beta\right) .
\end{aligned}
$$

The Jacobin matrix at the origin of system (37) can be written as follows:

$$
J_{(0,0)}^{2}=\left[\begin{array}{cc}
0 & -1 \\
1 & 0
\end{array}\right],
$$

and, with the help of computer algebraic system Maple, we can compute the first five Lyapunov constants for system (37) by using formal series method which was given in the following.

Theorem 3. For system (37), the first five Lyapunov constants at the origin can be given as follows: 


$$
\begin{aligned}
\mu_{2} & =\frac{i a_{5}\left(-2+\alpha \beta+\alpha^{2} \beta\right)}{\left.24 \alpha^{6}(1+\alpha)^{3} \beta^{2}\right)^{3}}\left(20 a_{5}^{2}-6 a_{5} \alpha \beta-4 a_{5}^{2} \alpha \beta-10 a_{5} \alpha^{2} \beta-4 a_{5}^{2} \alpha^{2} \beta+\alpha^{3} \beta^{2}+a_{5} \alpha^{3} \beta^{2}+\alpha^{4} \beta^{2}+a_{5} \alpha^{4} \beta^{2}-\alpha^{3} \beta^{3}\right. \\
& \left.-\alpha^{4} \beta^{3}\right), \\
\mu_{3} & =-\frac{i a_{5}\left(-2+\alpha \beta+\alpha^{2} \beta\right)}{1536 \alpha^{7}(1+\alpha)^{5} \beta(-5+\alpha(1+\alpha) \beta)^{3}}\left(a _ { 5 } \left(-240(12+5 \alpha(5+\alpha))-40 \alpha(1+\alpha)\left(-18+\alpha\left(79+57 \alpha+30 \alpha^{2}\right)\right) \beta\right.\right. \\
& +4 \alpha^{2}(1+\alpha)^{2}(480+\alpha(1126+\alpha(426+205 \alpha))) \beta^{2}-2 \alpha^{3}(1+\alpha)^{3}(96+\alpha(216+\alpha(54+73 \alpha))) \beta^{3}+2 \alpha^{4}(1+\alpha)^{4} \\
& \left.\cdot(-12+\alpha(-20+\alpha(3+10 \alpha))) \beta^{4}-\alpha^{6}(1+\alpha)^{5}\left(-4+\alpha^{2}\right) \beta^{5}\right)+\alpha^{2}(1+\alpha)(-1+\beta) \beta(-480+\alpha(1+\alpha) \\
& \left.\left.\cdot\left(200+\beta\left(720+\alpha\left(-420+\alpha\left(-360+(1+\alpha) \beta\left(204+154 \alpha-2(1+\alpha)(6+\alpha(2+7 \alpha)) \beta+\alpha^{3}(1+\alpha)^{2} \beta^{2}\right)\right)\right)\right)\right)\right)\right), \\
\mu_{4} & =\frac{i a_{5}(-1+\beta)\left(-2+\alpha \beta+\alpha^{2} \beta\right)}{46080 \alpha^{6}(1+\alpha)^{5}\left(-5+\alpha \beta+\alpha^{2} \beta\right)^{2} h_{6}} h_{4}, \\
\mu_{5} & =\frac{i a_{5}(-1+\beta)\left(-2+\alpha \beta+\alpha^{2} \beta\right)}{13271040 \alpha^{8}(1+\alpha)^{7}\left(-5+\alpha \beta+\alpha^{2} \beta\right)^{4} h_{6}} h_{5},
\end{aligned}
$$

where $h_{4}, h_{5}, h_{6}$ are given in the appendix.

Theorem 3 yields the following.

Proposition 4. For system (37), the first five Lyapunov constants at the origin are zero if and only if one of the following conditions holds:

$$
\begin{aligned}
& a_{1}=-\frac{-1+\alpha \beta+\alpha^{2} \beta}{1+\alpha}, \\
& a_{5}=0 ; \\
& a_{1}=-\frac{1+a_{5}+a_{5} \alpha}{1+\alpha}, \\
& \beta=\frac{2}{\alpha(1+\alpha)} .
\end{aligned}
$$

Furthermore, we have the following.

Theorem 5. The origin of system (37) is a center if and only if one of the conditions in Proposition 4 holds.

Proof. When condition (40) holds, system (37) becomes

$$
\begin{aligned}
& \frac{d u}{d t}=-\frac{v(u+\alpha)(1+u+\alpha)}{\alpha(1+\alpha)}, \\
& \frac{d v}{d t}=-\frac{u\left(-v+\alpha+\alpha^{2}\right)(-1+v \beta)}{\alpha(1+\alpha)},
\end{aligned}
$$

which has four invariant algebraic curves

$$
\begin{aligned}
& L_{1}=u+\alpha, \\
& L_{2}=u+1+\alpha, \\
& L_{3}=-v+\alpha+\alpha^{2}, \\
& L_{4}=-1+v \beta,
\end{aligned}
$$

and admits an integrating factor

$$
\begin{aligned}
& \mu_{4}(x, y) \\
& =L_{1}^{-1+\alpha \beta(-1+\alpha(1+\alpha) \beta)} L_{2}^{-1-(1+\alpha) \beta(-1+\alpha(1+\alpha) \beta)} L_{3}^{-1-\alpha(1+\alpha) \beta} .
\end{aligned}
$$

When condition (41) holds, system is simplified into

$$
\frac{d u}{d t}=-\frac{v(u+\alpha)(1+u+\alpha)}{\alpha(1+\alpha)},
$$

$\frac{d v}{d t}$

$$
=\frac{\left(-v+\alpha+\alpha^{2}\right)\left(-2 u v+a_{5} v^{2}+a_{5} u v^{2}+\alpha u+a_{5} \alpha v^{2}+\alpha^{2} u\right)}{\alpha^{2}(1+\alpha)^{2}},
$$

which has three invariant algebraic curves $L_{1}, L_{2}, L_{3}$ and admits an integrating factor

$$
\mu_{5}(x, y)=L_{1}^{1+2 a_{5}+2 /(1+\alpha)} L_{2}^{-(\alpha+2) / \alpha} L_{3}^{-3} .
$$


Two examples of phase plane of system (42) when condition (40) or (41) is satisfied are given in Figures 1 and 2.

Based on Theorem 3, we can conclude the following.

Theorem 6. The origin of system (37) is fifth order weak foci if and only if one of the following conditions holds:

$$
\begin{aligned}
H_{1}: & a_{1}=-0.2282, \\
a_{5} & =0.337573, \\
\alpha & =-9.39076168286412, \\
\beta & =0.02411227315630107 ;
\end{aligned}
$$

$$
\begin{aligned}
H_{2}: & a_{1}=-1.57238, \\
& a_{5}=0.688478, \\
\alpha & =4.0444054384945956, \\
\beta & =0.28989931115360074 ; \\
H_{3}: \quad a_{1} & =2.5, \\
a_{5} & =0, \\
\alpha & =-0.5000000000000074, \\
\beta & =0.9999999999999943 .
\end{aligned}
$$

Proof. Let $\mu_{1}=\mu_{2}=\mu_{3}=0$, and we obtain

$$
\begin{aligned}
& a_{1}=\frac{2 a_{5}-\alpha \beta-\alpha^{2} \beta+a 5 \alpha^{2} \beta+a 5 \alpha^{3} \beta+\alpha^{2} \beta^{2}+2 \alpha^{3} \beta^{2}+\alpha^{4} \beta^{2}}{\alpha(1+\alpha)^{2} \beta} \\
& a_{5}^{2}=\frac{-6 a_{5} \alpha \beta-10 a_{5} \alpha^{2} \beta+\alpha^{3} \beta^{2}+a_{5} \alpha^{3} \beta^{2}+\alpha^{4} \beta^{2}+a_{5} \alpha^{4} \beta^{2}-\alpha^{3} \beta^{3}-\alpha^{4} \beta^{3}}{4\left(-5+\alpha \beta+\alpha^{2} \beta\right)} \\
& a_{5}=\frac{\alpha^{2}(1+\alpha)(-1+\beta) \beta}{h_{6}} h_{4}
\end{aligned}
$$

submitting $a_{5}$ into $a_{5}^{2}$, we have

$$
\begin{aligned}
h_{7} & =-2880(2+\beta)+\alpha(-80(102+\alpha(74 \\
& +5 \alpha(5+\alpha)))+80(-249+\alpha(-412+\alpha(-216 \\
& \left.\left.\left.-66 \alpha+\alpha^{3}\right)\right)\right) \beta+4(1+\alpha)(-1260+\alpha(150 \\
& +\alpha(-2188 \\
& +\alpha(1576+\alpha(1295+\alpha(350+69 \alpha))))) \beta^{2} \\
& -4 \alpha(1+\alpha)^{2}(-1620+\alpha(-2730+\alpha(-3805 \\
& +2 \alpha(-526+\alpha(-79+\alpha(57+20 \alpha)))))) \beta^{3} \\
& +2 \alpha^{2}(1+\alpha)^{3}(684+\alpha(522+\alpha(244 \\
& +\alpha(-468+\alpha(-342+\alpha(24+19 \alpha)))))) \beta^{4} \\
& -2 \alpha^{3}(1+\alpha)^{4}(-36+\alpha(48+\alpha(110 \\
& +\alpha(-74+\alpha(-58+\alpha(5+3 \alpha)))))) \beta^{5}-\alpha^{5}(1 \\
& +\alpha)^{5}\left(12+14 \alpha+\alpha^{3}\right) \beta^{6}+\alpha^{7}(1+\alpha)^{6} \beta .
\end{aligned}
$$

Solve

$$
\begin{aligned}
& h_{4}=0, \\
& h_{7}=0
\end{aligned}
$$

and $h_{5} \neq 0$, and we can obtain the results.
Considering the reality, all parameters are positive in a predator-prey system, so we cannot get five limit cycles for this system.

In fact, $h_{5}=0$ means that

$$
\alpha=\frac{-110742+151145 \beta}{106746}
$$

and, submitting it into $a_{1}, a_{5}$, we get Figure 3 which shows that $a_{1}, a_{5}$ cannot be larger than zero simultaneously.

We can adjust the parameters to satisfy $\mu_{1}=\mu_{2}=0, \mu_{3} \neq$ 0 , and all parameters are positive. For example,

$$
\begin{aligned}
& a_{1}=0.380667, \\
& a_{2}=0.268514, \\
& a_{3}=0.735621, \\
& a_{4}=0.481486, \\
& a_{5}=0.009, \\
& a_{6}=0.10697 .
\end{aligned}
$$

Then three limit cycles can be bifurcated from the origin; namely, bistable phenomenon can occur for this system; this is not discussed in previous discussion. 


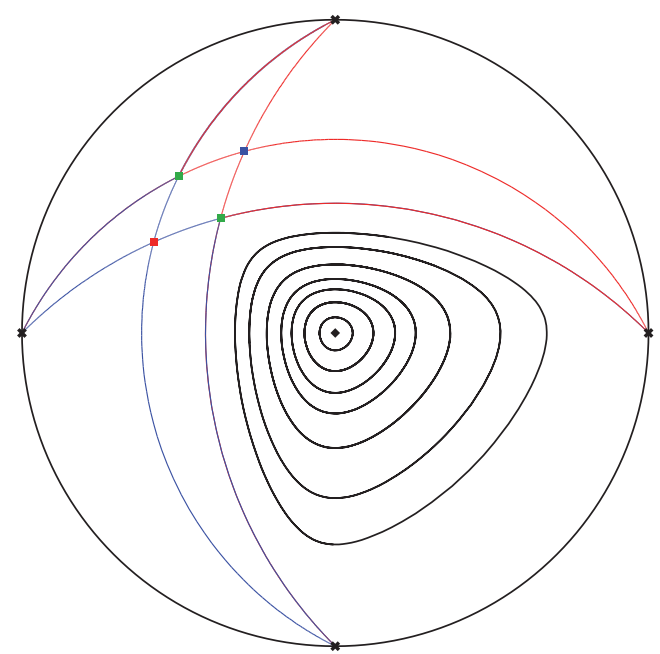

Figure 1: The phase plane of system (42) when $\alpha=\beta=1, a_{1}=$ $-1 / 2, a_{5}=0$.

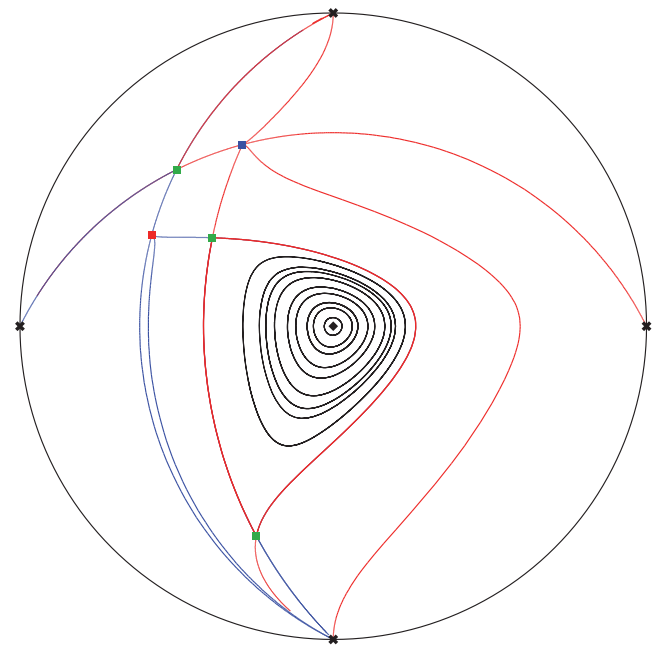

Figure 2: The phase plane of system (45) when $\alpha=\beta=1, a_{1}=$ $-3 / 2, a_{5}=1$.

\section{Boundary Singular Point}

In this section, we consider the boundary singular point of system (4). The boundary singular point is

$$
\left(u_{1}, 0\right)=\left(\frac{a_{2}+\sqrt{a_{2}^{2}+4 a_{1} a_{5}}}{2 a_{5}}, 0\right) \text {, }
$$

and the Jacobin matrix at $\left(u_{1}, 0\right)$ of system $(4)$ is

$$
\begin{aligned}
& J_{11}=-2 a_{1}-a_{2} u_{1}, \\
& J_{12}=-u_{1}\left(a_{3}+u_{1}\left(a_{4}+a_{5} u_{1}\right)\right), \\
& J_{21}=0, \\
& J_{22}=u_{1}-1 .
\end{aligned}
$$

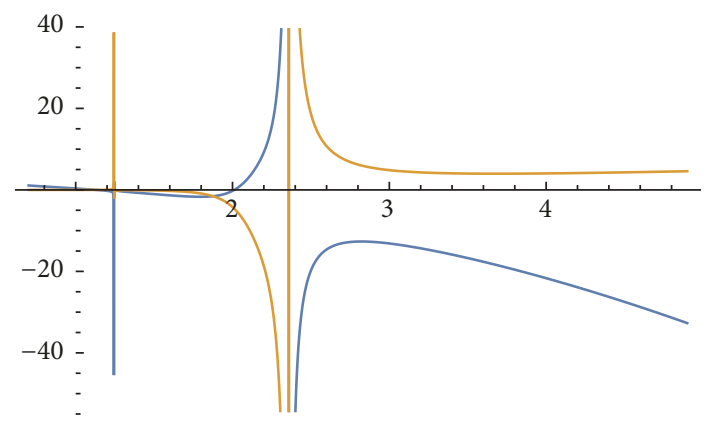

FIGURE 3: $a_{1}, a_{5}$ with $\beta$.

It is easy to testify that when $u_{1}>1$, system (4) has two eigenvalues with different signs which means $\left(u_{1}, 0\right)$ is a saddle. When $u_{1}<1$, system (4) has two eigenvalues with negative signs which means $\left(u_{1}, 0\right)$ is a stable node. When $u_{1}=1$, namely, $a_{5}=a_{1}+a_{2}\left(u_{1}, 0\right)$, system (4) becomes $(1,0)$ and one eigenvalue is zero. System (4) becomes

$$
\begin{aligned}
\frac{d u}{d t} & =u\left(a_{1}+a_{2} u-\left(a_{1}+a_{2}\right) u^{2}-a_{3} v-a_{4} u v\right. \\
& \left.-\left(a_{1}+a_{2}\right) u^{2} v-a_{6} v^{2}\right), \\
\frac{d v}{d t} & =v(u-1)(1+v) .
\end{aligned}
$$

Shifting the $(1,0)$ of system (4) to origin, system (4) becomes

$$
\begin{aligned}
\frac{d u}{d t} & =(1+u)\left(-v\left(a_{3}+a_{4}+a_{4} u+a_{6} v\right)\right. \\
& -a_{2}(1+u)(u+v+u v) \\
& \left.-a_{1}(v+u(2+u)(1+v))\right) \\
\frac{d v}{d t} & =v u(1+v)
\end{aligned}
$$

the Jacobin matrix at origin system (56) is

$$
\begin{aligned}
& J_{11}=-2 a_{1}-a_{2}, \\
& J_{12}=-a_{1}-a_{2}-a_{3}-a_{4}, \\
& J_{21}=0, \\
& J_{22}=0 .
\end{aligned}
$$

By transformation

$$
\begin{aligned}
& x=v, \\
& y=u+\frac{a_{1}+a_{2}+a_{3}+a_{4}}{2 a_{1}+a_{2}} v,
\end{aligned}
$$

and time scale 


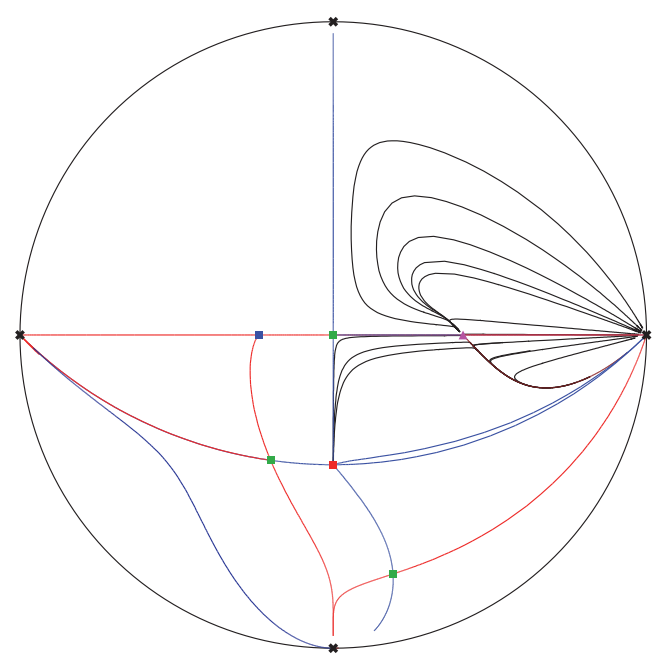

FIGURE 4: When $a_{1}=a_{2}=a_{3}=a_{4}=1$, the $(1,0)$ is a saddle node.

system (56) becomes

$$
\begin{aligned}
& \frac{d x}{d \sigma} \\
& \quad=\frac{x(1+x)\left(a_{1} x+a_{2} x+a_{3} x+a_{4} x-2 a_{1} y-a_{2} y\right)}{\left(2 a_{1}+a_{2}\right)^{2}} \\
& \quad=\phi(x, y), \\
& \frac{d y}{d \sigma}=y+\alpha_{2} x^{2}+\alpha_{3} x^{3}+\cdots=\psi(x, y) .
\end{aligned}
$$

Based on the theorem in [31], we have the following.

Theorem 7. The origin of system (56) is a saddle-node singular point.

Proof. From $\psi(x, y)=0$, we get a solution $y=\varphi(x)$ by implicit function theorem; submitting it into $\phi(x, y)$, we have

$$
\phi(x, y)=\frac{a_{1}+a_{2}+a_{3}+a_{4}}{\left(2 a_{1}+a_{2}\right)^{2}} x^{2}+\text { h.o.t. }
$$

Based on the theorem in [31], the origin of system (56) is a saddle-node singular point.

An numerical simulation of phase portrait of system (56) when $a_{1}=a_{2}=a_{3}=a_{4}=1$ is given in Figure 4 which shows that the origin of system (56) is a saddle-node singular point.

\section{Conclusion}

In this paper, a class of predator-prey systems with fear effect is investigated; the integrability conditions of $(0,0)$ and positive equilibrium are obtained and proved by finding their integrating factors. Furthermore, we compute the first five focal values at the positive equilibrium; considering the reality, when we choose all parameters to be positive, the fact that at least three limit cycles can be bifurcated from the positive equilibrium is proved. Consequently, bistable phenomenon can occur for this system.

\section{Appendix}

$$
\begin{aligned}
& h_{1}=18 a_{3}+123 a_{3}^{2}+330 a_{3}^{3}+435 a_{3}^{4}+282 a_{3}^{5}+72 a_{3}^{6} \\
& +10 a_{6} \\
& -a_{3} a_{6}-136 a_{3}^{2} a_{6}-305 a_{3}^{3} a_{6}-252 a_{3}^{4} a_{6}-72 a_{3}^{5} a_{6} \\
& \quad-34 a_{6}^{2} \\
& -122 a_{3} a_{6}^{2}-141 a_{3}^{2} a_{6}^{2}-54 a_{3}^{3} a_{6}^{2}-a_{3} a_{6}^{3} \\
& h_{2}=8928 a_{3}+125304 a_{3}^{2}+790932 a_{3}^{3}+2964090 a_{3}^{4} \\
& +7328577 a_{3}^{5}+12553050 a_{3}^{6}+15201327 a_{3}^{7} \\
& + \\
& + \\
& + \\
& +
\end{aligned}
$$




$$
\begin{aligned}
& +27474066 a_{3}^{16}+1612800 a_{6}+508864 a_{3} a_{6} \\
& -218871752 a_{3}^{2} a_{6}-2177749360 a_{3}^{3} a_{6} \\
& \text { - } 11040362722 a_{3}^{4} a_{6}-35943609534 a_{3}^{5} a_{6} \\
& -81875803362 a_{3}^{6} a_{6}-135933906721 a_{3}^{7} a_{6} \\
& \text { - } 167682085018 a_{3}^{8} a_{6}-154481849807 a_{3}^{9} a_{6} \\
& -105570924254 a_{3}^{10} a_{6}-52454569137 a_{3}^{11} a_{6} \\
& -18233779068 a_{3}^{12} a_{6}-4133758239 a_{3}^{13} a_{6} \\
& -532194624 a_{3}^{14} a_{6}-27474066 a_{3}^{15} a_{6} \\
& -15402880 a_{6}^{2}-158256544 a_{3} a_{6}^{2} \\
& -557121532 a_{3}^{2} a_{6}^{2}-11527076 a_{3}^{3} a_{6}^{2} \\
& +6684250599 a_{3}^{4} a_{6}^{2}+27220235893 a_{3}^{5} a_{6}^{2} \\
& +60677507163 a_{3}^{6} a_{6}^{2}+88881737479 a_{3}^{7} a_{6}^{2} \\
& +90150286040 a_{3}^{8} a_{6}^{2}+63931693138 a_{3}^{9} a_{6}^{2} \\
& +31132657395 a_{3}^{10} a_{6}^{2}+9899219979 a_{3}^{11} a_{6}^{2} \\
& +1841541615 a_{3}^{1} 2 a_{6}^{2}+150615531 a_{3}^{13} a_{6}^{2} \\
& +42920976 a_{6}^{3}+546275032 a_{3} a_{6}^{3} \\
& +3048202608 a_{3}^{2} a_{6}^{3}+9828708469 a_{3}^{3} a_{6}^{3} \\
& +20239699980 a_{3}^{4} a_{6}^{3}+27635538151 a_{3}^{5} a_{6}^{3} \\
& +24947109574 a_{3}^{6} a_{6}^{3}+14102138654 a_{3}^{7} a_{6}^{3} \\
& +4088412094 a_{3}^{8} a_{6}^{3}-66138543 a_{3}^{9} a_{6}^{3} \\
& -402713712 a_{3}^{1} 0 a_{6}^{3}-82890243 a_{3}^{11} a_{6}^{3} \\
& -11071544 a_{6}^{4}-280371244 a_{3} a_{6}^{4} \\
& -1959232014 a_{3}^{2} a_{6}^{4}-6805169344 a_{3}^{3} a_{6}^{4} \\
& -13984019309 a_{3}^{4} a_{6}^{4}-18183884031 a_{3}^{5} a_{6}^{4} \\
& -15196558244 a_{3}^{6} a_{6}^{4}-7941085336 a_{3}^{7} a_{6}^{4} \\
& -2367652521 a_{3}^{8} a_{6}^{4}-308023677 a_{3}^{9} a_{6}^{4}-68686928 a_{6}^{5} \\
& -526930142 a_{3} a_{6}^{5}-1719513766 a_{3}^{2} a_{6}^{5} \\
& -3098266867 a_{3}^{3} a_{6}^{5}-3332665876 a_{3}^{4} a_{6}^{5} \\
& -2141983550 a_{3}^{5} a_{6}^{5}-762239374 a_{3}^{6} a_{6}^{5} \\
& -115925697 a_{3}^{7} a_{6}^{5}+114184 a_{6}^{6}-977878 a_{3} a_{6}^{6} \\
& -5076266 a_{3}^{2} a_{6}^{6}-8132230 a_{3}^{3} a_{6}^{6}-5507306 a_{3}^{4} a_{6}^{6} \\
& -1358944 a_{3}^{5} a_{6}^{6}-1168 a_{6}^{7}+18464 a_{3} a_{6}^{7} \\
& +48136 a_{3}^{2} a_{6}^{7}+29152 a_{3}^{3} a_{6}^{7} ; \\
& h_{4}=-13282560-43642560 \alpha-49286720 \alpha^{2} \\
& -30272800 \alpha^{3}-9389600 \alpha^{4}-1680000 \alpha^{5} \\
& -6641280 \beta-68094240 \alpha \beta-199957120 \alpha^{2} \beta \\
& -239003040 \alpha^{3} \beta-134068800 \alpha^{4} \beta-46858320 \alpha^{5} \beta \\
& -6727040 \alpha^{6} \beta-711600 \alpha^{7} \beta-16495200 \alpha \beta^{2} \\
& -64422960 \alpha^{2} \beta^{2}-127913696 \alpha^{3} \beta^{2} \\
& -160881424 \alpha^{4} \beta^{2}-47151048 \alpha^{5} \beta^{2} \\
& +69526920 \alpha^{6} \beta^{2}+49186488 \alpha^{7} \beta^{2}+15788568 \alpha^{8} \beta^{2} \\
& +2384560 \alpha^{9} \beta^{2}+13194720 \alpha^{2} \beta^{3}+109470432 \alpha^{3} \beta^{3} \\
& +357478320 \alpha^{4} \beta^{3}+608912304 \alpha^{5} \beta^{3} \\
& +610603968 \alpha^{6} \beta^{3}+385444836 \alpha^{7} \beta^{3} \\
& +153076800 \alpha^{8} \beta^{3}+31586928 \alpha^{9} \beta^{3}+839520 \alpha^{10} \beta^{3} \\
& -221172 \alpha^{11} \beta^{3}+20857680 \alpha^{3} \beta^{4}+116720952 \alpha^{4} \beta^{4} \\
& +292955568 \alpha^{5} \beta^{4}+443927004 \alpha^{6} \beta^{4} \\
& +408517924 \alpha^{7} \beta^{4}+160896060 \alpha^{8} \beta^{4} \\
& -61488328 \alpha^{9} \beta^{4}-89757012 \alpha^{10} \beta^{4} \\
& -36079476 \alpha^{11} \beta^{4}-7970236 \alpha^{12} \beta^{4}-946600 \alpha^{13} \beta^{4} \\
& -8639856 \alpha^{4} \beta^{5}-65029176 \alpha^{5} \beta^{5}-224637196 \alpha^{6} \beta^{5} \\
& -469610888 \alpha^{7} \beta^{5}-643883792 \alpha^{8} \beta^{5} \\
& -581454748 \alpha^{9} \beta^{5}-331021368 \alpha^{10} \beta^{5} \\
& -104319138 \alpha^{11} \beta^{5}-6835256 \alpha^{12} \beta^{5} \\
& +8743616 \alpha^{13} \beta^{5}+3875660 \alpha^{14} \beta^{5}+528526 \alpha^{15} \beta^{5} \\
& -2156688 \alpha^{5} \beta^{6}-14976744 \alpha^{6} \beta^{6}-44914796 \alpha^{7} \beta^{6} \\
& -73132028 \alpha^{8} \beta^{6}-60367870 \alpha^{9} \beta^{6}+1849238 \alpha^{10} \beta^{6} \\
& +64720258 \alpha^{11} \beta^{6}+76234376 \alpha^{12} \beta^{6} \\
& +43548912 \alpha^{13} \beta^{6}+10939230 \alpha^{14} \beta^{6}-768234 \alpha^{15} \beta^{6} \\
& -992248 \alpha^{16} \beta^{6}-139758 \alpha^{17} \beta^{6}-119376 \alpha^{6} \beta^{7} \\
& -544344 \alpha^{7} \beta^{7}-40046 \alpha^{8} \beta^{7}+4865314 \alpha^{9} \beta^{7} \\
& +13867462 \alpha^{10} \beta^{7}+16901435 \alpha^{11} \beta^{7}+5715696 \alpha^{12} \beta^{7} \\
& -10216601 \alpha^{13} \beta^{7}-15072230 \alpha^{14} \beta^{7}-8697693 \alpha^{15} \beta^{7} \\
& -2005174 \alpha^{16} \beta^{7}+189135 \alpha^{17} \beta^{7}+172420 \alpha^{18} \beta^{7}
\end{aligned}
$$




$$
\begin{aligned}
& +21506 \alpha^{19} \beta^{7}+31344 \alpha^{8} \beta^{8}+259960 \alpha^{9} \beta^{8} \\
& +946607 \alpha^{10} \beta^{8}+2005159 \alpha^{11} \beta^{8}+2806319 \alpha^{12} \beta^{8} \\
& +2902527 \alpha^{13} \beta^{8}+2525365 \alpha^{14} \beta^{8}+1969903 \alpha^{15} \beta^{8} \\
& +1248297 \alpha^{16} \beta^{8}+527699 \alpha^{17} \beta^{8}+104568 \alpha^{18} \beta^{8} \\
& -9970 \alpha^{19} \beta^{8}-8164 \alpha^{20} \beta^{8}-942 \alpha^{21} \beta^{8}-3542 \alpha^{10} \beta^{9} \\
& -33124 \alpha^{11} \beta^{9}-137480 \alpha^{12} \beta^{9}-332573 \alpha^{13} \beta^{9} \\
& -517324 \alpha^{14} \beta^{9}-537908 \alpha^{15} \beta^{9}-376096 \alpha^{16} \beta^{9} \\
& -173390 \alpha^{17} \beta^{9}-50638 \alpha^{18} \beta^{9}-9184 \alpha^{19} \beta^{9} \\
& -1256 \alpha^{20} \beta^{9}-157 \alpha^{21} \beta^{9}+157 \alpha^{12} \beta^{10}+1413 \alpha^{13} \beta^{10} \\
& +5652 \alpha^{14} \beta^{10}+13188 \alpha^{15} \beta^{10}+19782 \alpha^{16} \beta^{10} \\
& +19782 \alpha^{17} \beta^{10}+13188 \alpha^{18} \beta^{10}+5652 \alpha^{19} \beta^{10} \\
& +1413 \alpha^{20} \beta^{10}+157 \alpha^{21} \beta^{10} ; \\
& h_{5}=-507810216960-2436546197760 \alpha \\
& -5096227659520 \alpha^{2}-6454790185600 \alpha^{3} \\
& -4937667216000 \alpha^{4}-2381071216000 \alpha^{5} \\
& -656298640000 \alpha^{6}-101282400000 \alpha^{7} \\
& -253905108480 \beta-3289813426560 \alpha \beta \\
& -12575546048000 \alpha^{2} \beta-22642178078720 \alpha^{3} \beta \\
& -23432213111040 \alpha^{4} \beta-14293863398720 \alpha^{5} \beta \\
& -4125897489920 \alpha^{6} \beta-260258558400 \alpha^{7} \beta \\
& +308399068800 \alpha^{8} \beta+55578240000 \alpha^{9} \beta \\
& -781865055360 \alpha \beta^{2}-5108953531200 \alpha^{2} \beta^{2} \\
& -13363899231360 \alpha^{3} \beta^{2}-13734522948480 \alpha^{4} \beta^{2} \\
& +4744206996640 \alpha^{5} \beta^{2}+24102546471840 \alpha^{6} \beta^{2} \\
& +26859360388320 \alpha^{7} \beta^{2}+17174786811040 \alpha^{8} \beta^{2} \\
& +6082585707200 \alpha^{9} \beta^{2}+1257508167040 \alpha^{10} \beta^{2} \\
& +150976164800 \alpha^{11} \beta^{2}-29462497920 \alpha^{2} \beta^{3} \\
& +2081290798080 \alpha^{3} \beta^{3}+12656752005120 \alpha^{4} \beta^{3} \\
& +34903669038560 \alpha^{5} \beta^{3}+62279353510560 \alpha^{6} \beta^{3} \\
& +71285331244240 \alpha^{7} \beta^{3}+44514953713120 \alpha^{8} \beta^{3} \\
& +8875751706000 \alpha^{9} \beta^{3}-5301134386400 \alpha^{10} \beta^{3} \\
& -4181778690000 \alpha^{11} \beta^{3}-1319823350560 \alpha^{12} \beta^{3} \\
& -163625102960 \alpha^{13} \beta^{3}+830505487680 \alpha^{3} \beta^{4} \\
& +6026573491680 \alpha^{4} \beta^{4}+16813440957408 \alpha^{5} \beta^{4} \\
& +17834441035488 \alpha^{6} \beta^{4}-12873240733584 \alpha^{7} \beta^{4} \\
& -62936499854640 \alpha^{8} \beta^{4}-89549706151720 \alpha^{9} \beta^{4} \\
& -75036327882312 \alpha^{10} \beta^{4}-40825891602752 \alpha^{11} \beta^{4} \\
& -13640251694224 \alpha^{12} \beta^{4}-2215634370840 \alpha^{13} \beta^{4} \\
& +5554753200 \alpha^{15} \beta^{4}+491100684480 \alpha^{4} \beta^{5} \\
& +2633085796704 \alpha^{5} \beta^{5}+4903469917296 \alpha^{6} \beta^{5} \\
& +766891893632 \alpha^{7} \beta^{5}-14958638903792 \alpha^{8} \beta^{5} \\
& -62906756600 \alpha^{14} \beta^{4}-34094437668592 \alpha^{9} \beta^{5} \\
& -33147090532560 \alpha^{10} \beta^{5}-3433078824676 \alpha^{11} \beta^{5} \\
& +25876949348632 \alpha^{12} \beta^{5}+28176338678548 \alpha^{13} \beta^{5} \\
& +14438182246928 \alpha^{14} \beta^{5}+4269444571076 \alpha^{15} \beta^{5} \\
& +791830445928 \alpha^{16} \beta^{5}+77558760220 \alpha^{17} \beta^{5} \\
& +46717862400 \alpha^{5} \beta^{6}+857379648192 \alpha^{6} \beta^{6} \\
& +6256152709632 \alpha^{7} \beta^{6}+25914858817384 \alpha^{8} \beta^{6} \\
& +69454497287528 \alpha^{9} \beta^{6}+127173964745564 \alpha^{10} \beta^{6} \\
& +161521971496276 \alpha^{11} \beta^{6}+140047250437968 \alpha^{12} \beta^{6} \\
& +77684057329756 \alpha^{13} \beta^{6}+21422475391368 \alpha^{14} \beta^{6} \\
& -3187908175996 \alpha^{15} \beta^{6}-5595351902616 \alpha^{16} \beta^{6} \\
& -2471859280404 \alpha^{17} \beta^{6}-574519294148 \alpha^{18} \beta^{6} \\
& -57571385480 \alpha^{19} \beta^{6}-299527741440 \alpha^{6} \beta^{7} \\
& -2783409813120 \alpha^{7} \beta^{7}-12267246396328 \alpha^{8} \beta^{7} \\
& -34518869803248 \alpha^{9} \beta^{7}-69961743965804 \alpha^{10} \beta^{7} \\
& -108325367563788 \alpha^{11} \beta^{7}-131833660935316 \alpha^{12} \beta^{7} \\
& -127342881444188 \alpha^{13} \beta^{7}-96758400588528 \alpha^{14} \beta^{7} \\
& -55723515416210 \alpha^{15} \beta^{7}-22145087456212 \alpha^{16} \beta^{7} \\
& -4407388612126 \alpha^{17} \beta^{7}+769899797780 \alpha^{18} \beta^{7} \\
& +804052648670 \alpha^{19} \beta^{7}+220349460504 \alpha^{20} \beta^{7} \\
& +21962178666 \alpha^{21} \beta^{7}-10364564160 \alpha^{7} \beta^{8} \\
& -63393528864 \alpha^{8} \beta^{8}-74434095200 \alpha^{9} \beta^{8} \\
& +553845369068 \alpha^{10} \beta^{8}+3038953452776 \alpha^{11} \beta^{8}
\end{aligned}
$$




$$
\begin{aligned}
& +8370441701964 \alpha^{12} \beta^{8}+16173443952294 \alpha^{13} \beta^{8} \\
& +24259376312110 \alpha^{14} \beta^{8}+28873491631404 \alpha^{15} \beta^{8} \\
& +26633736985694 \alpha^{16} \beta^{8}+18132908433638 \alpha^{17} \beta^{8} \\
& +8420336551126 \alpha^{18} \beta^{8}+2205732470850 \alpha^{19} \beta^{8} \\
& +31126246206 \alpha^{20} \beta^{8}-183762333012 \alpha^{21} \beta^{8} \\
& -54793322296 \alpha^{22} \beta^{8}-5292633582 \alpha^{23} \beta^{8} \\
& +6303950784 \alpha^{8} \beta^{9}+71063475936 \alpha^{9} \beta^{9} \\
& +373839316928 \alpha^{10} \beta^{9}+1211118188320 \alpha^{11} \beta^{9} \\
& +2662686755878 \alpha^{12} \beta^{9}+4062227064942 \alpha^{13} \beta^{9} \\
& +4035457380180 \alpha^{14} \beta^{9}+1656683678325 \alpha^{15} \beta^{9} \\
& -2218674821968 \alpha^{16} \beta^{9}-5259864025928 \alpha^{17} \beta^{9} \\
& -5723602586392 \alpha^{18} \beta^{9}-3988066841946 \alpha^{19} \beta^{9} \\
& -1807586988714 \alpha^{20} \beta^{9}-460334512678 \alpha^{21} \beta^{9} \\
& -229943307102 \alpha^{19} \beta^{11}-1920938866124 \alpha^{20} \beta^{11} \\
& -139539158738 \alpha^{21} \beta^{11}-86365251276 \alpha^{22} \beta^{11} \\
& -43145090554 \alpha^{23} \beta^{11}-15826247756 \alpha^{24} \beta^{11} \\
& -12307772716 \alpha^{22} \beta^{9}+31525713085 \alpha^{23} \beta^{9} \\
& -
\end{aligned}
$$

$$
\begin{aligned}
& -3479497878 \alpha^{25} \beta^{11}-77652804 \alpha^{26} \beta^{11} \\
& +197175462 \alpha^{27} \beta^{11}+51730068 \alpha^{28} \beta^{11} \\
& +4289050 \alpha^{29} \beta^{11}-787968 \alpha^{12} \beta^{12} \\
& +5522528 \alpha^{13} \beta^{12}+143182140 \alpha^{14} \beta^{12} \\
& +1003568364 \alpha^{15} \beta^{12}+3970302664 \alpha^{16} \beta^{12} \\
& +10496607766 \alpha^{17} \beta^{12}+20014835420 \alpha^{18} \beta^{12} \\
& +28748690926 \alpha^{19} \beta^{12}+32008459412 \alpha^{20} \beta^{12} \\
& +28222020442 \alpha^{21} \beta^{12}+20064009416 \alpha^{22} \beta^{12} \\
& +11678667666 \alpha^{23} \beta^{12}+5610580272 \alpha^{24} \beta^{12} \\
& +2198339002 \alpha^{25} \beta^{12}+663873052 \alpha^{26} \beta^{12} \\
& +130403282 \alpha^{27} \beta^{12}+5542324 \alpha^{28} \beta^{12} \\
& -5056714 \alpha^{29} \beta^{12}-1339196 \alpha^{30} \beta^{12} \\
& + \\
& +105726 \alpha^{31} \beta^{12}+114912 \alpha^{14} \beta^{13} \\
& + \\
& + \\
& +
\end{aligned}
$$




$$
\begin{aligned}
& -820 \alpha^{7} \beta^{2}+192 \alpha^{3} \beta^{3}+1008 \alpha^{4} \beta^{3}+1980 \alpha^{5} \beta^{3} \\
& +1958 \alpha^{6} \beta^{3}+1194 \alpha^{7} \beta^{3}+546 \alpha^{8} \beta^{3}+146 \alpha^{9} \beta^{3} \\
& +24 \alpha^{4} \beta^{4}+136 \alpha^{5} \beta^{4}+298 \alpha^{6} \beta^{4}+292 \alpha^{7} \beta^{4} \\
& +68 \alpha^{8} \beta^{4}-104 \alpha^{9} \beta^{4}-86 \alpha^{10} \beta^{4}-20 \alpha^{11} \beta^{4} \\
& -4 \alpha^{6} \beta^{5}-20 \alpha^{7} \beta^{5}-39 \alpha^{8} \beta^{5}-35 \alpha^{9} \beta^{5} \\
& -10 \alpha^{1} 0 \beta^{5}+6 \alpha^{11} \beta^{5}+5 \alpha^{12} \beta^{5}+\alpha^{13} \beta^{5} .
\end{aligned}
$$

\section{Data Availability}

No data were used to support this study.

\section{Conflicts of Interest}

The authors declare that they have no conflicts of interest.

\section{Authors' Contributions}

Both authors contributed equally to the writing of this paper and read and approved the final manuscript.

\section{Acknowledgments}

We are thankful for the support of Shandong Provincial Natural Science Foundation, China (No. ZR2014FL017).

\section{References}

[1] C. S. Holling, "The functional response of predators to prey density and itsrole inmimicry and population regulation," Memoirs of the Entomological Society of Canada, vol. 97, no. S45, pp. 5-60, 1965.

[2] R. M. May, "Limit cycles in predator-prey communities," Science, vol. 177, no. 4052, pp. 900-902, 1972.

[3] G. Seo and D. L. DeAngelis, "A predator-prey model with a holling type I functional response including a predator mutual interference," Journal of Nonlinear Science, vol. 21, no. 6, pp. 811833, 2011.

[4] J. Huang, S. Ruan, and J. Song, "Bifurcations in a predatorprey system of Leslie type with generalized Holling type III functional response," Journal of Differential Equations, vol. 257, no. 6, pp. 1721-1752, 2014.

[5] H. Zhu, S. A. Campbell, and G. S. Wolkowicz, "Bifurcation analysis of a predator-prey system with nonmonotonic functional response," SIAM Journal on Applied Mathematics, vol. 63, no. 2, pp. 636-682, 2003.

[6] S. Ruan and D. Xiao, "Global analysis in a predator-prey system with nonmonotonic functional response," SIAM Journal on Applied Mathematics, vol. 61, no. 4, pp. 1445-1472, 2001.

[7] H. I. Freedman and G. S. K. Wolkowicz, "Predator-prey systems with group defence: the paradox of enrichment revisited," Bulletin of Mathematical Biology, vol. 48, no. 5-6, pp. 493-508, 1986.
[8] G. S. K. Wolkowicz, "Bifurcation analysis of a predator-prey system involving group defence," SIAM Journal on Applied Mathematics, vol. 48, no. 3, pp. 592-606, 1988.

[9] R. S. Cantrell and C. Cosner, "On the dynamics of predator-prey models with the Beddington-DeAngelis functional response," Journal of Mathematical Analysis and Applications, vol. 257, no. 1, pp. 206-222, 2001.

[10] J. R. Beddington, "Mutual interference between parasites or predat ors and its effect on searching efficiency," Journal of Animal Ecology, vol. 44, no. 1, pp. 331-340, 1975.

[11] D. L. DeAngelis, A. H. Goldstein, and R. V. O’Neill, "A model for trophic interaction," Ecology, vol. 56, no. 4, pp. 881-892, 1975.

[12] T. W. Hwang, "Global analysis of the predator-prey system with Beddington-DeAngelis functional response," Journal of Mathematical Analysis and Applications, vol. 281, no. 1, pp. 395401, 2003.

[13] T. W. Hwang, "Uniqueness of limit cycles of the predatorprey system with Beddington-DeAngelis functional response," Journal of Mathematical Analysis and Applications, vol. 290, no. 1, pp. 113-122, 2004.

[14] Y. Song and X. Zou, "Bifurcation analysis of a diffusive ratiodependent predator-prey model," Nonlinear Dynamics, vol. 78, no. 1, pp. 49-70, 2014.

[15] Y. Song and X. Zou, "Spatiotemporal dynamics in a diffusive ratio-dependent predator-prey model near a Hopf-Turing bifurcation point," Computers \& Mathematics with Applications, vol. 67, no. 10, pp. 1978-1997, 2014.

[16] X. Wang, L. Zanette, and X. Zou, "Modelling the fear effect in predator-prey interactions," Journal of Mathematical Biology, vol. 73, no. 5, pp. 1179-1204, 2016.

[17] P. Aguirre, E. González-Olivares, and E. E. Sáez, “Three limit cycles in a Leslie-Gower predator-prey model with additive Allee effect," SIAM Journal on Applied Mathematics, vol. 69, no. 5, pp. 1244-1262, 2009.

[18] C. Li and H. Zhu, "Canard cycles for predator-prey systems with Holling types of functional response," Journal of Differential Equations, vol. 254, no. 2, pp. 879-910, 2013.

[19] E. González-Olivares and A. Rojas-Palma, "Multiple limit cycles in a Gause type predator-prey model with Holling type III functional response and Allee effect on prey," Bulletin of Mathematical Biology, vol. 73, no. 6, pp. 1378-1397, 2011.

[20] D. Xiao and H. Zhu, "Multiple focus and hopf bifurcations in a predator-prey system with nonmonotonic functional response," SIAM Journal on Applied Mathematics, vol. 66, no. 3, pp. 802819, 2006.

[21] E. González-Olivares, A. Rojas-Palma, and B. González-Yañez, "Multiple limit cycles in a Leslie-Gower-type predator-prey model considering weak Allee effect on prey," Nonlinear Analysis: Modelling and Control, vol. 22, no. 3, pp. 347-365, 2017.

[22] M. Han, L. Sheng, and X. Zhang, "Bifurcation theory for finitely smooth planar autonomous differential systems," Journal of Differential Equations, vol. 264, no. 5, pp. 3596-3618, 2018.

[23] M. Han, X. Hou, L. Sheng, and C. Wang, "Theory of rotated equations and applications to a population model," Discrete and Continuous Dynamical Systems - Series A, vol. 38, no. 4, pp. 21712185, 2018.

[24] M. Han, T. Petek, and V. G. Romanovski, "Reversibility in polynomial systems of ODE's," Applied Mathematics and Computation, vol. 338, pp. 55-71, 2018.

[25] P. Yu, M. Han, and J. Li, "An improvement on the number of limit cycles bifurcating from a nondegenerate center of 
homogeneous polynomial systems," International Journal of Bifurcation and Chaos, vol. 28, no. 6, Article ID 1850078, 2018.

[26] Y. Liu and F. Li, "Double bifurcation of nilpotent focus," International Journal of Bifurcation and Chaos, vol. 25, no. 3, Article ID 1550036, p. 10, 2015.

[27] F. Li and M. Wang, "Bifurcation of limit cycles in a quintic system with ten parameters," Nonlinear Dynamics, vol. 71, no. 1-2, pp. 213-222, 2013.

[28] T. Liu, Y. Liu, and F. Li, "A kind of bifurcation of limit cycles from a nilpotent critical point," Journal of Applied Analysis and Computation, vol. 8, no. 1, pp. 10-18, 2018.

[29] Y. Yan, Y. Pan, F. Lu, and Z. Zhou, "On the integrability and equivalence of the abel equation and some polynomial equations," Journal of Nonlinear Modeling and Analysis, vol. 1, no. 2, pp. 207-220, 2019.

[30] F. Li, Y. Liu, Y. Liu, and P. Yu, "Bi-center problem and bifurcation of limit cycles from nilpotent singular points in Z2-equivariant cubic vector fields," Journal of Differential Equations, vol. 265, no. 10, pp. 4965-4992, 2018.

[31] Z. Zhang, T. Ding, W. Huang, and Z. Dong, The Qualitative Theory of Differential Equations, Science Press, Bejing, China, 1997. 


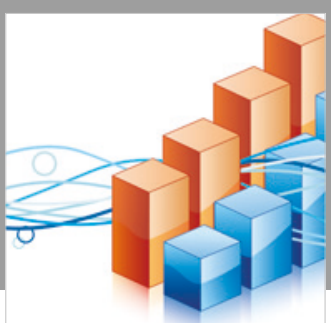

Advances in

Operations Research

\section{-n-m}
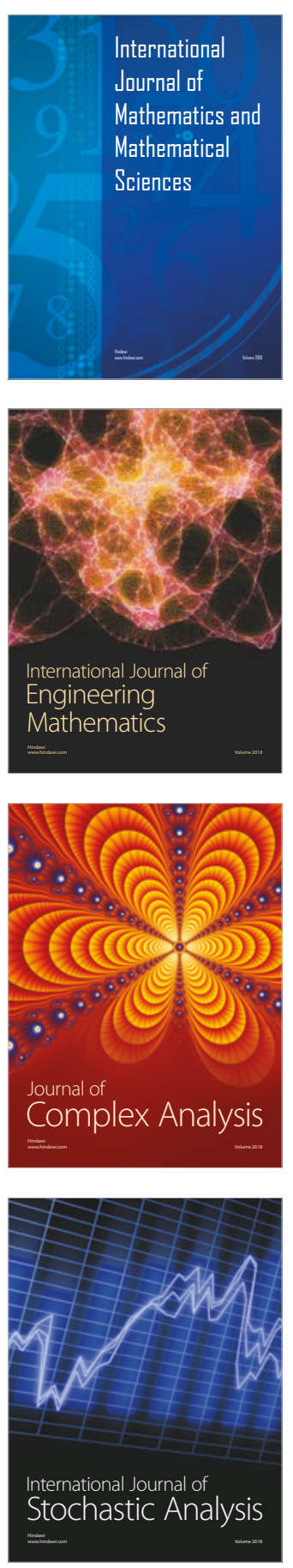
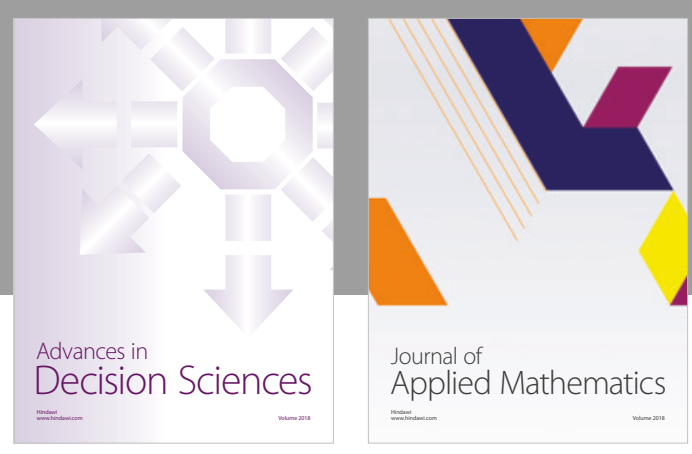

Journal of

Applied Mathematics
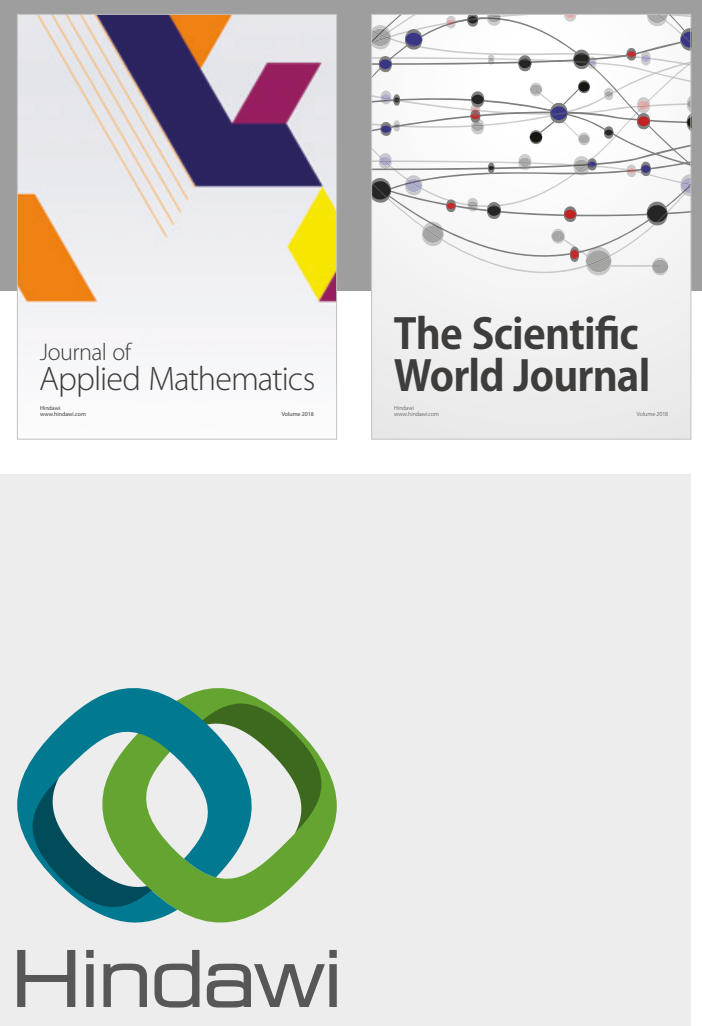

Submit your manuscripts at

www.hindawi.com

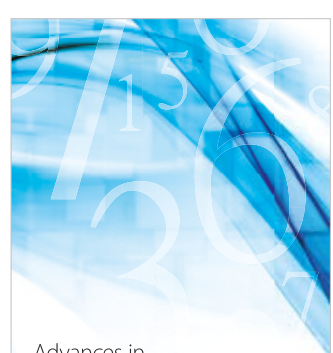

Advances in
Numerical Analysis
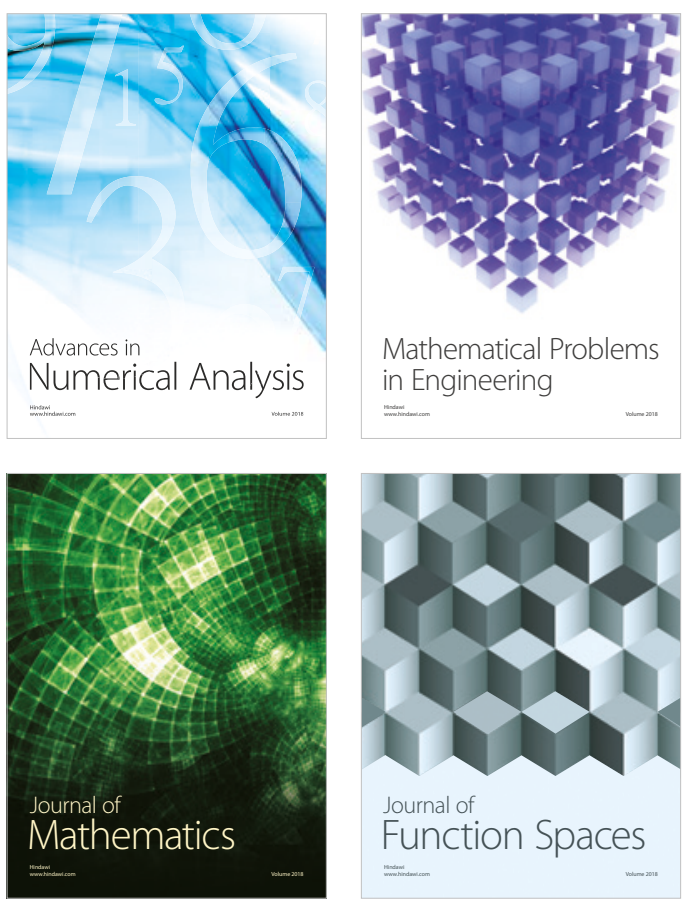

Mathematical Problems in Engineering

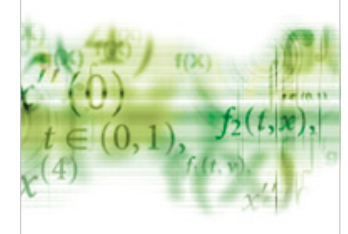

International Journal of

Differential Equations

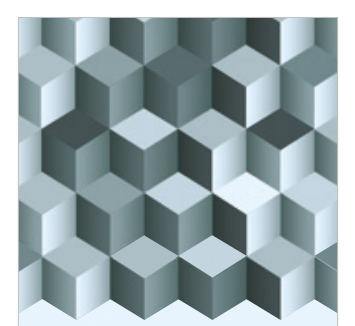

Journal of

Function Spaces
The Scientific

World Journal

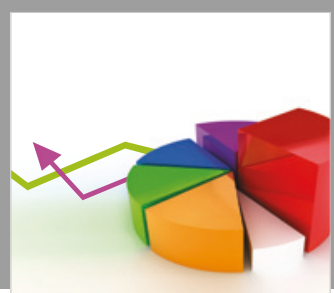

Journal of

Probability and Statistics
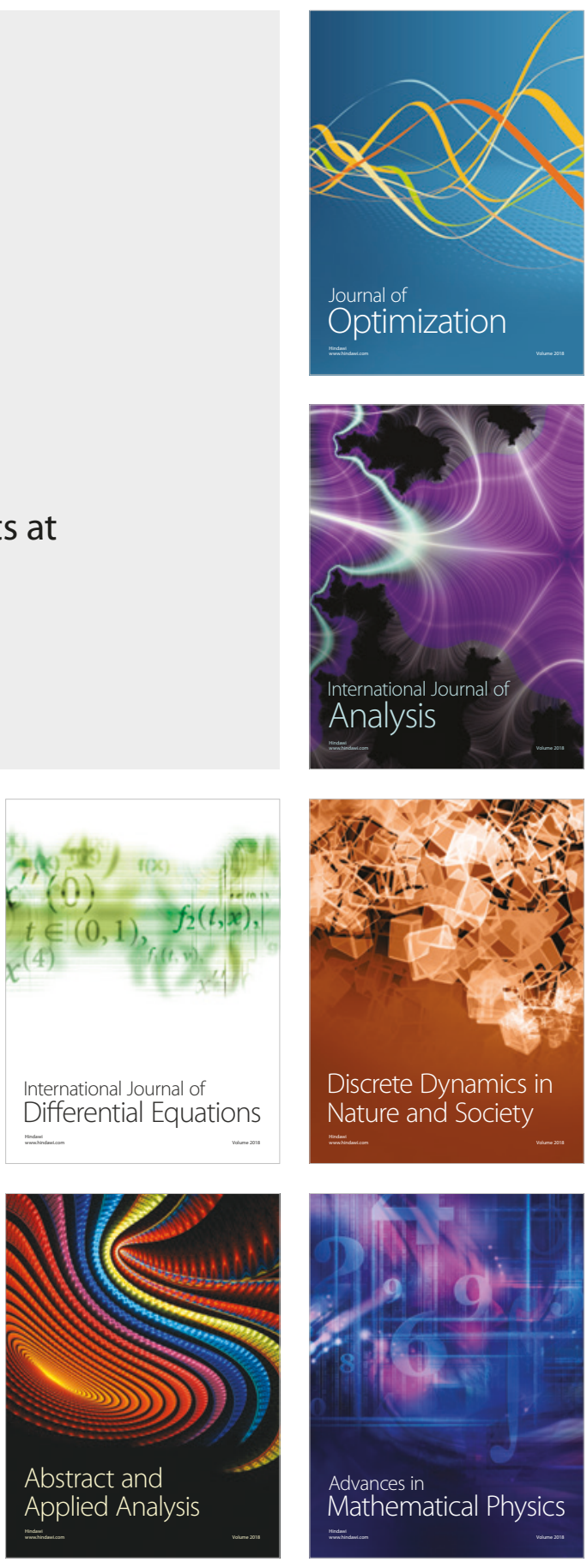